Veröffentlichungen treten rein. wissenschaftliche über die, soweit sie einschlägig und wichtig sind, in den Naturwissenschaften regelmäBig berichtet wird aufifallemd zurück gegenüber angewandt wissenschaftlichen. In erster Reihe stehen die augenblicklichen politischen Zustände, denen eine Flut von Aufsätzen, Büchern, Karten und selbst mehrbändigen Werken gewidimet wird. Dann folgen Schriften, die sich mit den vom Kriege herbeigeführten Veränderungen der Weltwirtschaft und - eine Folge des Weltrohistoffhungers - mit den Wirtschaftsquellen großer Gebiete, vornehmlich Amerikas, mit ihren äußersten Möglichkeiten und thit den Mitteln ihrer vollen Ausbeutung beschäftigen. Eine gewaltige Zunahme werist die militärgeographische Literatur gegenüber 1914 auf. - Im ganzen steht das geographische Leben stark unter dem Einflusse der Kriegs-. und Nachkriegsereignisse, und es ' hat nicht den Ansohein, als ob es sobald wieder in die fruchtbringendere Bahn unvoreingenommener rein wissenschaftilicher Tätigkeit zurückkehren wird. Im Gegenteil erweck' die genannte Literatur d'en Eindruck, als ob man sich auf künftige Ereignisse einzustellen, sich auch wissenschaftlich zu moibilisieren sucht. Ein Aufsatz Dryers (Märzheft S: 205), in dem vom kommenden "real Armageiddon", dem wahren Weltkriege, und von der ausschlaggebenden Rolle Amerikas in diesem Kampfe, ,der Kinder des Lichtes" gegen die „von Deutschland bis Japan“ arganisierten "Mächte der Finsternis", des "barbarischen Kernes der alten Welt" die Rede ist, und andererseits eine gewisse, schlẹcht verborgene Feindiseligkeit gegen England (Gibbons im Februarheft S. 145) deuten an, welchen Vorstellungen man sioh in dieser Hinsicht hingibt. Die Beziehungen zur deutschen Wissenschaft sind insafern wieder angeknüpft, als deutsche Literaturerscheinungen sachliche Würdigung erfahren. Im übrigen aber macht sich eine feindselige Tonart noch immer häufig genug geltend. Wie schon das Beispiel Dryers lehrt, steigert sie sich bisweilen zu Beschimpfungen - zum Schaden des Ansehens der amerikanischen Wissenschaft.

\section{B. Brandt.}

Die sächsischen Erdbeben während der Jahre 1907 bis 1915 hat F. Etzold im 36. Bd.d. Abhdlgen. d. math.phys. Kl. der sächs. Ges. ıd. Wissienisch. 1919 einer umfangreichen Darstellung unterzogen (S. 217-428). Innerbalb der genannten Zeit sind in Sachsen an 106 Tagen Erdbeben gefühlt worden. Die sächsischen Erderschütterungen sind zunächst von $H$. Credner besonders bearbeitet woridien, $C$, hat zur intensiveren Beobachtung eine Erdbebenkommission ins Leben gerufen. Die Anteilnahme der Bevölkerung in Sachsen an den Erdbebenbeobachtungen ist sehr groB. Für die Schütterperiode im Herbst 1908 wurde z. B. ein Beobachtungsmaterial gesammelt, das einen Zettelkatalog von 5370 Einzelzetteln rumfaßte. Nach Credners Tod hat Etzold diese Bebenbearbeitung übernommen.

In der vorliegenden Arbeit werden in möglichst großem Umfang die Originallbeobachtungen mitgeteilt, was leider nicht oft geschieht, so daß meist der Leser von der Auffassunig idess Bearbeitens der Beobachtungen abhängig wird. Anschließend an gröBerie Beobachtungsgruppen bringt der Verfasser ferner Betrachtungen, die zur Geologie des' Beobachtungsgebietes in Bezug stehen: Vor allem treten hierbei die Beziehungen zwischen beobachteter Bebenstärke und der geologisohen Beschaffenheit des betreffenden Geibietes hervor; besonders macht er auf die Wirkungen des Untergrundes und der Dislokationen aufmerksam. Solche Beziehun- gen sind dem Seismologen zwar bekannt und nicht überraschiend, in dem vorhandenen Beobachtungsmaterial sind aber doch oft besonders' auffallende Tatsachen bemerkbar. 'Auch idurch das sưdddeutsohe Beben aim 16. November 1911 ist ganz Sachsen den Menschen fühlbar erschüttert wonden, und zwar stärker ,als jemals durch die am Ort ihrer Entstehung Furcht und Schrecken erregenden vogtländischen Erdistöße". Nach dem Verfasser ist von besonderer Bedleutung der Umstand, daß in Sachsen kurz vor und nach dem HauptstoB am 16. November 1911 Endistöße beobachtet worden sind, die aber nicht mit den in Sïddeutschland beobachteten Vor-: undl Nachibeben zeitlich zusammenfallen, „mit gröBter Wahrscheinlichkeit ist aber ein kausaler Zusammenhang zwischen dem siidldeutsichen Hauptsto $\beta$ und zum mindiesiten den aus idem chronischen vogtländischen Schüittergebiet, also aus Adorf und Asch, gemeldeten Erdstößen anzunehmen, es dürften insbesondere tdiejenigen; welche sich dort nach dem 16. November um $23^{\mathrm{h}}$ ereigmeten, als durch jenen Hauptstoß $\beta$ ausgelöst żu igelten haben“".

Von ganz besonderem Interesse ist der Abschnitt "Seismogenetissche Erörterungen", in denen er zunächsit iiber eigene Erdbebenbeobachtungen berichtet, eine Charakteristik der vogtländischen Erdistöße gibt und im letzten Unterabschnitt zur Frage iber Wesen und Ursache der vogtländiischen Erdibeben Stelliung nimmt. Auf Grund eigener Beobachtungen bezeichnet Etzold die vogtländisoh-erzgebingischen Erderschütterungen als Enscheinungen, „die unter lautem Geräusch auf Linien fortschrieiten und beiderseits ider letzteren den Boden in rasche Zittierbewiegungen versetzen". Dieser lineare Verlauf der Erschitterungen ist nach Etzold für die Erkenntnis ihres Wesens und ihrer Ursache von größter B̉edeutung. Bei jiediem vogtländischen Schwarmbeben denken bekanintlich Laien an vulkanische Beben und weisen auf bevonstehende Ausbrüche der Vulkane Kammerbühl und Eisenbühll hin. Nach den bisherigen Beobachtungen ist aber bei solchen Beben die in fühllbarer Weise enschütterte Fläche nicht sehr groß. Z. B. wurde durch die Explosion des Bandaisan 1888 nur ein Areal von 5000. qkm erschüttert, demgegenüber steht dile gelegentlich der vagtländlischen Beben den Menschen fühlbar bewegte Fläche von $40000 \mathrm{qkm}$.

Schon bei der ersten Publikation über dlie vogtländischen Erdstöße ist. 1876 H. Credner für deren tektonische Natur eingetreten, die „zu erlklären sein dürften als Äußerungen der Gebirgsentstiehuing und unterirdischen "Spaltenbildungt in Folge seitlichen Drucks". Später, auf Grund weiterer Beobachtungen, fügte Creidner seiner Auffassung ergänzende Erklärungen zu; es scheint, diaß er sich ïber ddie Ursächlichkeit der vogtländischen Beben nicht recht schlüssig werden konnte. Gümbel, Becke, Knett, Th. Brandes, Koßmat, Lohrmann, Jacobi nehmen als Ursache diesser Beben tektonische Vorgänge an. Dieser Erklärung tritt Etzold nicht bei und hält eine andere Anschauung für die richtigere.

Nach, Etzolds eigenen Beobachtungen machten sich die vogtländischen Erderschüitterungen im Herbst 1908 im Epizentralgebilet ,durch rasiches Fortschreiten auf einer Linie, dureh den rasselnden, sich gleichsam überstürzenden Donner und dürch die rüttelnden Bodenbewegungen" auf and zu Seiten der Propagationslinile"-bemerkbar. „Danach kann man bei diesem seismischen Phänomen nur an einen Spaltenauf:reißungsprozeB bzw, an dien Beginn eines solchen den- 
ken und wind als nächstverwandte Eracheinungen an die Bergschläge sowie an das knallende und schreiende Gebirge erinnert". In den folgeniden Zeilen führt Etzold entsprechend der Bergschlatge und venwandte Erscheinungen thehanddelnden Aribeit von Rzehak mehrere Bejspiele an, die an den Verlauf der vogtländischen Erdbeben erinnern, und auch Rzohak bemerkt in der Ztsehr. f. prakt. Geologie 1906, ,daB die Bergschläge zü einem der wichtigsten geódynamischen Phänomene hinleiten, nämlich zu den-,tektonisichen" Erdbeben". Als Ursache für 'idie Bengsiehläge werden auf verschiedene Weisen zustande gekommene Spannungen angenommen, deren Auslösung ,zu einer Riß- odier Spaltenbildung unter mehr oder minder lautiem, krachendem Geräusch" führt. In "Kohle und Erz" 1920 beschäftigt sich J. Schlesiona in zwei Auf́sätzen mit ahnlliohen in Kòhlenflözen auftetetenden ispannungen. Für das Auftreten bergschlagartiger. Ersicheinungen ist festes gesundes Gestein Vorbedingrung. Etzold bringt noch weitere Erhebungen, die seine Annahme stützen. Einige von thm angefiuhrte Erfahrungen hat Referent gelegentlich der vogtländischen Erdbeben 1908 dort auch selbst gemacht. Zur weiteren Klärung der Frage iiber das Entstehen dier genannten Beben stellt $E$. ein Arbeitsprogramm auf.

Mainka.

Neue Polarlichtforsehungen in Norwegen. · Seitdem der Franzose A. Bravais vor acht Jahrzehnten seine klassischen Studien ther das Nordlicht zu Bossekop im norwegischen Finnmarken angestellt hat, ist dieser Ort ein Dorado fiur Nordlichtiorschungen geworden. Insbesondere ist es schon 1910 Professor Carl störmer in Kristiania gelungen, zahlreiche Photographien dieser eigenartigen Lichterscheinung in Bossekop aufzunehmen und die photogrammetrische Methode zur Bestimmung ihrer Höhe anzuwenden. Diese Arbeiten hat der unermuldliche Polarliohtforscher weiterhin noeh in $\mathrm{Kri}$ stiania fortgesetzt; er hat ein außerordentlich reichhaltiges photographisches Material zusammengebracht, dessen wertvollster Teil aus pairweisen Aufnahmen besteht, die gleichzeitig an den Enden von Standlinien aufgenommen wurden, deren Länge 27 bis $100 \mathrm{~km}$ beträgt, ⿶0 daB die Genauigkeit der Höhenbestimmungen auBerordentlich grob ist. Wir entwehmen den neuesten Veröffentlichungen störmers ${ }^{1}$ ) folgende Einzelheiten, die von allgemeinem İnteresse sein dürften.

Ein am 4. Oktober 1919 bei Kristiania photographierter NordlichtstrahI reichte von 140 bis $\mathrm{zu} 410 \mathrm{~km}$ Höhe, hatte also eine Mindestlänge voni $270 \mathrm{~km}$. $\mathbf{E r}$ stand im Zenit einer mehr als $300 \mathrm{~km}$ weiter nordnordösthich gelegenen Gegend des mittleren Schweden. Eine am 17. Oktober 1919 axfigenommene Draperie reichte von 100 bis $270 \mathrm{~km}$ Höhe and befand sich $550 \mathrm{~km}$ nördlich von Kristiania.

Besonders reiche Ausbeute jedoch lieferte das große Nordlicht vom 22. bis 23. März 1920, das in groben Teilen der nördlichen Hallkkagel, $z_{*} B$. in Paris, Nord-

1) Situation, dans l'espace de quelques aurores boréales. Bulletin ide la Societé Astronomique de France. Paris 1920, avril. 7 pag. 3 diagrammes; 6 photographies, 1 carte. - L'Aurore Boréale le 22-23 Mars 1920. Astronomische Nachrichten, 1920, Band 211, Juni, Nr. 5047, Spalte $131-136$. - Sur quelques royous auroraux observes le 22 mans 1920 at at teignant l'altitude de $500 \mathrm{~km}$. Comptes rendus de l'Acadiemie des Sciences, Paris, 1920, Tome 171, Séance du 13 septembre, 1 pag. - Nogle fotografier af Nordlyskronen om mongtenen dien 23. marts 1920 taget frä Bygdo ved Kristiania Nordisk Astronomisk Tidskrift, Koben havn, 1920, Bind 1, No. 4. 4 pag, 6 fotografier. amenika usw, gesehen wurde. Gieben norwegische Stationen, die telephonisch miteinander in Verbindung. standien and 26 bis $224 \mathrm{~km}$ voneinandier entfernt lagen, liaferten in neunstundiger Arbeit 620 Photagraphien, von denen 73 an zwleri, 50 sogar an drei Stationen gleichzeitig aufgenommen waren. Ihre Auswertung ergab als uberraschendes Resultat Maximalhöben der Strahlen von der GröBenordnung $500 \mathrm{~km}$. Eine große Tahl dieser Strahlen bildeten eine sogenannte Nordlichtkrone, d. h. sia vereinigten sich scheinibar im magnetischen Zenit, so dab es gelang, auch die Position dieses Radiationspunktes mit groBer Genauigkeit zil bestimmen. In seinen Veröffentlichungen reproduziert Störmer seebs Photographien solcher Nordlichtkronen, die natürlich nicht imstande sind, einen Begriff von der grandiosen Pracht dieser selfmen Naturerscheinung zu geben, derlen unbeschreibliche Schömheit ausdriicklich hervorgehoben wird. Die Strahlen, welche die Krone bildeten, hatten eine blane bis violette Farbe.

Die normale gelbgrine Spektrallinie fand Störmer bei der Nordlichtkrone nur. schwach entwickelt und wenig hervertretend. Diese Beobachtung ditrfte jedoch auf eine subjektive Beeinflussung durch die überatus hellen Linien in den anderen Teilen des Spektrums zurückzuführen sein, die ich schon 1892 bei Nordlichtkronen häufig mit großer Intensität aufblitzen ader längere Zeit verweilen sehen konnte2): Stormer sah ebenfalls eine Menge Spektrallinien, jedoch vornehmllich in dem blamen und violetten Teil ides Spektrums, die ibm auf Wasserstoff oder Helium za deuten scheinen.

Bemerkenswert ist die Tatsachẹ, daß zú dem gleichen Termin, an dem die bláuien Strahlén der Nordlichtkrone bei Kristiania auftraten, das Nordlicht auch in den Vereinigten Staaten von Amerika, wo es an mehr als hundert Stationen beobachtet wurde, seine größte Intensität erreichte. $\quad$ O. Baschin.

Asthma, Heuschnupfen und verwandte Erscheinungen. Francis M. Rackemann, Med. elin. of NorthAmerice Bd. 3, Nr. 4, S, 1065-1076, 1920.) Heuschnupfen, Asthma, Urticaria und neben diesen drei wohlcharakteriksierten Typen anch noch einige Manifestationen des Magendarmkanalis (gewisse Formen von Erbrechen, Leibschmerzen) und der Haut, ferner dias angioneurotische Odem sind in den detzten Jahren allgemein (amerikanische und englische Literatur) als Zeichen einer Uberempfindlichlseit gegen bestimmte Eiwreifarten, als anaphylaktischa Reaktion angesehen wordien. Die Grundiagen dieser Ansicht beruhen anf drei Tatsachren. 1. Die genannten Erkrankungsformèn stehen in getsetzmäßitigen Beziehungen zu Proteilnkörpern, mit denten Erkrankte durch Einatmung, Nahrungszuifuhr usw, in Berührung kommen. 2. Die gletichen Proteinkörper bewirken nach Stich oder Auftröpfelung eine, heftigge Haut- bzw. Schleimhantreaktion 3. Mehrfache Injektionen dieser fremden Eiweißsubstanzen bringen die klinisehen Erscheinungen zum Schwinden. - Bei Betrachtung der Krankheitissymptome, in Anbetracht der minimalen Mengen Eiwolb, dia schon Enscheinungen veranlasisen, erinnert die Sensibilisilerung der Asthmatiker, Heufieberkranken usw. gegen gewisise Eiweibsorten von selbst an die Anaphylaxsie des Meerschweinchens. Jedoch be-

2) Otto Baschin, Die ersten Nordlichtphotographien, aufgenommen in Bossekop (Lappland). Meteorologische Zieitschrift, Wien, 1900 , Bd. $1 \%$, S. 278-280. Tafel IV. 